\title{
Dressing the Scalar Glueball
}

\author{
M. Boglione and M.R. Pennington \\ Centre for Particle Theory, University of Durham, \\ Durham DH1 3LE, U.K.
}

\begin{abstract}
The hadronic dressing of the ten lightest scalar mesons is discussed.
\end{abstract}
Let us imagine that we live in a world without quarks. QCD suggests there will still be a spectrum of hadrons, bound states of two or more gluons, the lightest of these being stable. Though this world is far from that explored by experiment, it is the world of the lattice calculator. There the lightest state is a scalar in the region of $1-2 \mathrm{GeV}$, just where we expect the lowest $q \bar{q}$ scalars to be.

The lattice makes definite predictions for the bare glueball mass, depending on the calculational scheme : $1550 \mathrm{MeV}$ from UKQCD (1), $1600 \mathrm{MeV}$ from Morningstar and Peardon (2) and $1740 \mathrm{MeV}$ from the GF-11 group at IBM (3). Though this state is stable in the quenched world of the lattice, the IBM group have pioneered the calculation of its coupling to 2 pseudoscalar sources and found this would correspond to a width of some $100 \mathrm{MeV}$ (4). Naively, one would expect the bare glueball to couple as an $S U(3)_{F}$ singlet. The IBM group have calculated that in fact coupling to heavier pseudoscalars should be favoured (4). Thus the lattice makes predictions for the scalar gluestate with some element of choice about its exact mass and coupling scheme. Here we will focus on just one for ease of presentation. We will take a mass of $1600 \mathrm{MeV}$ with a coupling pattern as given by the IBM group (note that this pattern is not very sensitive to the exact glueball mass). However, very similar results apply for other choices.

Of course, the bare non-decaying state is not what any experiment can observe. A bare state, whether composed of glue or quarks, has to be dressed by interactions with mesons in order to decay. Thus, though we like to think of the $\rho$ or $\phi$ as $q \bar{q}$ states, their Fock space contains important $\pi \pi$ and $K \bar{K}$ components, respectively, through which these hadrons decay. For most mesons, and in particular, the vectors and tensors, these hadronic dressings make rather little difference to the states. So the hadrons we observe and the underlying $q \bar{q}$ bound states are simply related and readily identifiable one from the other. However, as has been emphasised by Tornqvist (5), this is not the case for scalars. This is because their couplings are larger and because, in as much as decays to two pseudoscalars dominate, their interactions are $S$-wave making the opening of the 
corresponding thresholds especially important, as Bugg (6) has long stressed.

While for vectors and tensors, decays can be reliably modelled by the ${ }^{3} P_{0}$ mechanism, this is known to fail for scalars $(7,8)$. The Schwinger-Dyson equation for the scalar propagators is the natural vehicle for calculating these necessarily non-perturbative effects. In the self-energy loops (Fig. 1) one must sum over all hadronic intermediate states, of these two pseudoscalars are the most important and the ones we discuss first $(9,10)$.

FIGURE 1. The bare bound state propagator is dressed by hadronic interactions. The dot signifies the dressed hadron propagator. The wiggly lines on the particles in the loop is to emphasize these too are bound states.

We start with the hadronic interactions turned off and consider the ten lightest scalars : the bare glueball and an ideally mixed $q \bar{q}$ nonet. While the lattice provides a definite statement about the mass and coupling of the gluestate, we have no such prediction for the bare $q \bar{q}$ nonet. We do know that the strange quark adds $\sim 100 \mathrm{MeV}$ to the mass, so we have just 2 parameters, the central mass of the $q \bar{q}$ multiplet and its coupling strength to two pseudoscalars. These will be fixed by the observed hadron spectrum as we shall see. By imposing the constraints of chiral symmetry on the scalar- 2 pseudoscalar coupling and by assuming it to have a form-factor reflecting a roughly common spatial extent of $0.7 \mathrm{fm}$ for mesons, we can compute, using the Schwinger-Dyson equation, the effect of dressing on the 10 bare states.

We begin with the $I=1 / 2$ sector, where the LASS experiment $(11,12)$ has pinned down the $K_{0}^{*}$ parameters close to those given in Table 1 . To get these right, the bare $n \bar{s}$ state is at $1520 \mathrm{MeV}$. The large $K \pi$ and $K \eta^{\prime}$ couplings provide the decays of the dressed hadron and shifts its mass to $1420 \mathrm{MeV}$. Though this result is very similar to that of Tornqvist (9), our calculation differs in an important respect. Here only propagators are computed, these determine the right hand cut structure of the corresponding hadronic amplitudes - the $D$-function in $N / D$. In contrast, Tornqvist enforces a much more restrictive range of parameters by requiring that the $N$-function is wholly real and fitting data on the hadronic scattering amplitudes. This is a drastic oversimplification. It assumes that amplitudes contain only $s$-channel dynamics. Of course, in reality the $N-$ function has a left hand cut, the structure of which is determined by crossed channel dynamics that Tornqvist simply ignores.

With the parameters of our calculation essentially determined by the $K_{0}^{*}$-pole position, we turn to the $I=1$ sector. The bare $n \bar{n}$ state is at $1420 \mathrm{MeV}$, but the 
very strong dressing by the $\pi \eta$ and $K \bar{K}$ channels draws the hadron pole towards $K \bar{K}$ threshold generating an $a_{0}(980)$ with a Fock space containing only $20 \% n \bar{n}$, but $70 \% K \bar{K}$. The $I=0$ sector has 3 bare states : $n \bar{n}, s \bar{s}$ and $g g$. These mix through their common hadronic channels $\pi \pi, K \bar{K}, \eta \eta, \eta \eta^{\prime}$ and $\eta^{\prime} \eta^{\prime}$. The physical scalars are the eigenstates of the resulting mass matrix. Again states are markedly shifted : the $f_{0}(980)$ is drawn to $K \bar{K}$ threshold - see Table 1. Importantly, just as noted by Tornqvist $(9)$, the $a_{0}(980)$ and the $f_{0}(980)$ are automatically the dressed partners of the $K_{0}^{*}(1430)$. This is in stark contrast to the model of Janssen et al. (13) where the $a_{0}$ and $f_{0}$ are quite unconnected or the first order perturbative mixing scheme of Amsler and Close (14) or Weingarten (15) where the $a_{0}(980)$ and $f_{0}(980)$ have to be additional states totally unrelated to the $K_{0}^{*}(1430)$. Our broad isoscalar is the $f_{0}(1300)$ (12) (Table 1). In contrast, Roos and Tornqvist (16) claim a $\sigma(550)$ in their more restrictive scheme, which as already mentioned violates crossing symmetry (17). Our third $f_{0}$ is closely related to the bare gluestate. A common outcome of the bare coupling schemes we have considered is that the mixing suppresses this hadron's couplings to 2 pseudoscalars reducing its bare $100 \mathrm{MeV}$ pseudoscalar width to $25 \mathrm{MeV}$ or so (10). Whether the bare gluestate is at 1600 or $1740 \mathrm{MeV}$, the hadron's branching fraction to $K \bar{K}$ is always reduced and that to $\eta \eta$ is strongest (10). This makes identification with the $f_{J}(1710) \mathrm{MeV}$ rather unlikely $(18,19,12)$.

TABLE 1. Masses and widths of the ten lightest scalars given by their pole positions in $\mathrm{MeV}$ and their branching ratios to 2 light pseudoscalars from the calculation of Ref. 10.

\begin{tabular}{rccc}
\hline Resonance & $m_{\text {pole }}$ & $\Gamma_{\text {pole }}$ & BR(PS) \\
\hline$K_{0}^{*}(1430)$ & 1445 & 334 & $100 \%$ \\
$a_{0}(980)$ & 1082 & 309 & $100 \%$ \\
$f_{0}(980)$ & 1006 & 54 & $100 \%$ \\
$f_{0}(1300)$ & 1203 & 361 & $100 \%$ \\
$f_{0}(1550)^{\dagger}$ & 1564 & 108 & $23 \%$ \\
\hline
\end{tabular}

$\dagger$ Here the $f_{0}(1550)$ results from a bare gluestate chosen to be at $1600 \mathrm{MeV}(2,10)$.

Of course, 2 pseudoscalars are not the only channels, multipion modes are inevitably important. As the lattice gives no idea of the strength of these, we fix the bare gluestate coupling to these wholly phenomenologically by requiring an output hadron with a total width of $\sim 100 \mathrm{MeV}$, where as before $25 \mathrm{MeV}$ or less is in 2 pseudoscalar channels. The larger bare coupling shifts the mass a little more, so that a bare mass of $1600 \mathrm{MeV}$ moves down to $1564 \mathrm{MeV}$ (Table 1).

The identification of the state predicted here with what is seen in experiment requires a careful analysis of a range of production processes, not just $\bar{p} p$ annihilation at rest (20) or $J / \psi$ radiative decay (18). Only by the consistent analysis of many channels produced in different ways (18-23) can one arrive at a meaningful set of parameters for the tenth scalar. Such analyses have started $(24,25)$. 
However, it should be remembered that while experiment determines the dressed states (these being the poles of the $S$-matrix) with reasonable accuracy, the poles of the $K$-matrix, which are the underlying states (Fig. 1 ), are not fixed unambiguously. Different solutions with differing numbers of $K$-matrix poles, but very similar $S$-matrix poles, may describe the data equally well. Thus the underlying poles are not uniquely determinable from data with present accuracy and so the true bare states are not easily found, despite (25). Hence our predictions

for the dressed states is what matters. With these, we should soon know if the $f_{0}(1500)(20-23,12)$ is the predominantly glue candidate or not.

\section{REFERENCES}

1. G.S. Bali et al. (UKQCD), Phys. Lett. B389, 378 (1993).

2. C. Morningstar and M. Peardon, Nucl. Phys. Proc. Suppl. 53, 917 (1997).

3. J. Sexton et al., Phys. Rev. Lett. 75, 4563 (1995).

4. J. Sexton et al., presented at "Lattice 95", Melbourne, Australia, Jul 1995, hep-lat/9602022.

5. N.A. Tornqvist, Acta Phys. Pol. B16, 503 (1985).

6. D.V. Bugg, Proc. Hadron '89, Ajaccio (ed. F. Binon et al), Editions Frontiers, p. 567.

7. P. Geiger and N. Isgur, Phys. Rev. D44, 799 (1991); D47, 5050 (1993).

8. E.S. Ackleh et al., Phys. Rev. 54, 6811 (1996).

9. N.A. Tornqvist, Z. Phys. C68, 647 (1995).

10. M. Boglione and M.R. Pennington, Phys. Rev. Lett. 79, 1998 (1997).

11. D. Aston et al., Nucl. Phys. B296, 493 (1988).

12. R.M. Barnett et al., Review of Particle Physics, Phys. Rev. D 54, 30 (1996).

13. G. Janssen et al., Phys Rev. D52, 2690 (1995).

14. C. Amsler, F.E. Close, Phys. Rev. D53, 295 (1996); Phys.Lett. B353, 385 (1995).

15. D. Weingarten, Nucl. Phys. Proc. Suppl. 53 (1997) 232.

16. N.A. Tornqvist, M. Roos, Phys. Rev. Lett. 76, 1575(1996).

17. N. Isgur and J. Speth, Phys. Rev. Lett. 77, 2332 (1996); M. Harada et al., Phys. Rev. Lett. 78, 1603 (1997).

18. J.E. Augustin et al., Z. Phys. C36, 369 (1987); Phys. Rev. Lett. 60, 2238 (1988); R.M. Baltrusaitis et al., Phys. Rev. D35, 2077 (1987).

19. S.J. Lindenbaum et al., Phys. Lett. B274, 492 (1992).

20. C. Amsler et al., Phys. Lett. B342, 433 (1995); B353, 571 (1995); B355, 425 (1995).

21. F. Binon et al., Nuovo Cim. 80, 363 (1984); D. Alde et al., Phys. Lett. B201, 160 (1988).

22. T.A. Armstrong et al., Phys. Lett. B228, 536 (1989).

23. K. Peters and Ch. Strassburger, contributions to these Proceedings.

24. E.g. A. Sarantsev, Proc. Hadron '95, Manchester, (ed. M. Birse et al.), World Sci., p. 384. 25. V.V. Anisovich, contribution to these Proceedings. 